\title{
La directividad en la relación terapéutica: análisis de la evidencia encontrada en investigación
}

\section{Directivity in the therapeutic relationship: analysis of the evidence found in research}

\author{
Alfonso Urzúa \\ Escuela de Psicología, Universidad Católica del Norte, Chile \\ Karim Arbach, Margarita Lourido, Natália Mas \\ Facultad de Psicología, Universidad de Barcelona, España
}

(Rec: 17 agosto 2009 / Acep: 15 marzo 2010)

\begin{abstract}
Resumen
Se expone los resultados de una revisión de las propuestas teóricas y hallazgos empíricos de diferentes autores e investigaciones sobre la directividad en la psicoterapia. En algunos casos la literatura localizada confluye en conceptos comunes y en otros diverge en sus resultados llevando a conclusiones heterogéneas sobre la directividad como habilidad terapéutica. Se presenta la información en capítulos tomando como eje central las perspectivas sobre cuándo ser más o menos directivos. En cada capítulo se desarrolla teóricamente el tema y a continuación los estudios que prestan o no soporte empírico a la propuesta teórica. Los contenidos posteriormente son sintetizados en la conclusión. Finalmente, la discusión integra los conceptos de mayor relevancia, analiza las limitaciones de los estudios y de los resultados encontrados y propone futuras líneas de investigación.
\end{abstract}

Palabras clave: Directividad, habilidad terapéutica, relación terapéutica

\begin{abstract}
One exposes the results of a revision of the theoretical proposals and empirical findings of different authors and investigations on the directivity in the psycotherapy. In some cases located Literature comes together in common concepts and in others it diverges in its results taking to heterogenous conclusions on the directivity like therapeutic ability. The information in chapters appears more or less taking like central axis the perspective on when being directors. In each chapter it is theoretically developed to the subject and next the studies that render or empirical nonsupport to the theoretical proposal. The contents later are synthesized in the conclusion. Finally, the Integra discussion the concepts of greater relevance, it analyzes the limitations of the studies and the found results and proposes future lines of investigation.
\end{abstract}

Key words: Directivity, therapeutic ability, therapeutic relation. 


\section{Introducción}

A pesar de que en la última década han disminuido algunas brechas en el conocimiento de factores que influyen en la relación terapéutica, como por ejemplo el reconocimiento de factores no específicos que intervienen en la efectividad de la psicoterapia, como las características, actitudes y competencias comunicacionales del terapeuta, así como la resistencia al cambio y su tratamiento, aún resalta la necesidad de estudios coordinados y metodológicamente rigurosos que seleccionen, especifiquen y prioricen la totalidad de variables del paciente y psicoterapeuta que conduzcan con mayor probabilidad a resultados positivos $\mathrm{y}$ consistentes.

Una de las características del terapeuta que se ha investigado es la directividad, en donde diversos investigadores intentan explicar cómo esta característica puede influir en la efectividad de la terapia, en la adherencia al tratamiento o incluso en la intención de buscar ayuda profesional (Beutler, 1991).

A fin de analizar los hallazgos sobre directividad, se efectúa una revisión teórica de las investigaciones publicadas entre los años 1990 y agosto del 2007 incorporadas a las bases de datos Psychinfo y artículos previos solamente cuando eran frecuentemente citados en investigaciones más recientes.

Dado que la bibliografía que aborda específicamente la directividad como objetivo de estudio no es extensa, a que usualmente las investigaciones enfocan esta habilidad terapéutica desde diferentes ópticas y que además, existe cierta variabilidad en lo que cada autor propone como definición de conducta directiva, se ha sistematizado la información en los siguientes apartados: (a) Aspectos conceptuales sobre directividad; aborda las convergencias y discrepancias en la conceptualización de la directividad, así como en su evaluación, (b) directividad e intervención psicoterapéutica: incluye los estudios que comparan distintos tipos de intervenciones como los que estudian en profundidad las modalidades de interacción en una terapia particular y en las distintas fases en que la misma se desarrolla y (c) directividad como característica particular del terapeuta $y$ del paciente : involucra aquellos estudios referidos a las características demográficas (edad, sexo) y de personalidad de quien aplica la intervención, así como las investigaciones que indican de qué modo los clientes de acuerdo a ciertos atributos personales, sea nivel de reactancia, locus de control (han sido los más investigados), condición psicopatológica, características demográficas (edad, sexo o etnia) perciben a su terapeuta o bien qué factores sociodemográficos se han relacionado con directividad.

\section{Aspectos conceptuales sobre directividad}

A la hora de delimitar una definición única e integradora con relación a directividad, ocurre que dependiendo del estudio, de los fines que persiga, de los métodos por la cual se evalúa, y obviamente, del autor que la plantea, surgen diversas y variadas definiciones tales como "conductas de enseñar y de confrontar" (Patterson \& Forgatch, 1985 en Bischoff y Tracey, 1995) o el "grado en que se dan instrucciones, se proporciona información, se ofrece ayuda específica, se estructuran y delimitan tareas" (Bados y García, 2001). Para Bischoff y Tracey (1995) es “(a) cualquier intento de liderar, dirigir o controlar la actividad verbal en la terapia y (b) cualquier declaración de desafío o confrontación al cliente." Y conductas directivas serían enseñar, estructurar, confrontar o desafiar, preguntar o informar directivamente, interpretar directivamente; Blau (1988, en Kleinke, 1995), define la directividad del terapeuta a lo largo de un continuo que iría desde (a) No intrusivo: se ofrece aceptación, empatía y estímulo, mediante metáforas y analogías (b)Moderadamente intrusivo: se intenta clarificar y desafiar las ideas del cliente y establecer límites cuando parezcan apropiados y (c)Probativo: se realizan interpretaciones y se utilizan actividades dirigidas como rol-playing, imaginación y expresión conductual; Cormier \& Cormier (1994), definen directividad como una característica de las respuestas de acción, las cuales incluyen datos y percepciones generados por el terapeuta; Schaap, Bennun, Schindler \& Hoogduin (1993), plantean que la categoría "control y directividad" contiene aquellas actividades del terapeuta que animan (alientan, estimulan) al cliente a exhibir y practicar comportamientos particulares tanto durante como fuera de la sesión e incluye instrucciones, consejos y tareas.

A pesar de que las mismas no resultan unívocas, pueden extraerse a partir de ellas algunos elementos comunes, por ejemplo indicadores relativamente acordados de la directividad del terapeuta tales como (a) Dar instrucciones. (b) Proveer información (c) Dar consejos y ayuda específicos (e) Estructurar y delimitar tareas (f) Mantener una postura activa e influyente.

Las diversas definiciones propuestas por los investigadores varían fundamentalmente en la delimitación de las conductas que incluyen como parte del repertorio asociado a la directividad. En un intento de integrar las características de mayor relevancia del término se propone que en el marco de las habilidades terapéuticas la directividad puede ser definida como aquellas intervenciones del terapeuta que, intencionadamente o no, en una sesión intentan dirigir, influir o modelar las respuestas naturales o el actuar del paciente, tanto dentro como fuera de ésta.

La definición precedente acota la directividad a las intervenciones (consejos, instrucciones, directivas) exclusivamente del terapeuta, independientemente que el paciente tienda a ser más o menos directivo; dichas intervenciones pueden ser intencionadas o no, ya que existe la posibilidad de que la directividad sea un "rasgo" de personalidad o una forma particular de interacción del terapeuta no necesariamente conciente para este; en una sesión terapéutica, 
se refiere a que las intervenciones se aplican en función del contexto particular de una sesión terapéutica más que en función del proceso terapéutico completo; tanto dentro como fuera de la sesión, pues lo que como finalidad última se busca es que la influencia del terapeuta traspase los límites temporales y espaciales de la consulta, impactando en la conducta intersesión del paciente, a fin de alcanzar un valor ecológico de los aprendizajes de la terapia.

\section{Evaluación de la Directividad}

El no existir unicidad de criterios al momento de definir la directividad se ha traducido en disímiles maneras de evaluarla. En la literatura revisada se ha encontrado que para evaluar ésta se han utilizado diferentes subescalas de pruebas más globales en donde la directividad es solo una dimensión a evaluar (Akutsu, Lin \& Zane, 1990; Bachelor \& Salame, 2000; Bennun, Hahlweg, Schindler \& Langlotz, 1986; Bischoff y Tracey, 1995; Fisher et al., 1995 en Karmo, Beutler y Harwood, 2002; Navarro, Schindlery \& Silva, 1987a,b; Shields \& McDaniel, 1992), no siendo posible localizar una prueba única que midiese específicamente directividad. Pareciera ser que existen conductas claramente observables y mensurables que los autores consideran como directivas y las incorporan en sus mediciones, otras son discutidas y utilizadas dependiendo del autor y por último otras contempladas en rara ocasión, y que a juicio de los autores de este trabajo, no presentan una clara vinculación teórica con la directividad (tabla 1).

En términos generales, las conductas en las que pareciera haber mayor consenso al evaluar directividad pueden ser agrupadas en las siguientes dimensiones:

a) Estructuración: grado de estructuración de entrevista y organización global del proceso y la intervención psicoterapéutica. b) Influencia: direccionamiento del cliente, presentación de opiniones "poderosas", modelamiento de la conducta, asignación de tareas.

c) Comunicación directiva: uso de interrogantes específicos, consejo y retroalimentación.

d) Conducta Activa: actuación autoritaria y decidida.

\section{Directividad e intervención terapéutica}

\section{Tipo de Terapia}

La comparación empírica del uso de directividad en el marco de modelos específicos de intervención ha llamado bastante la atención a los investigadores.

Desde que se ha comprobado y reconocido que los pacientes consideran que la relación con el terapeuta puede ser de mayor ayuda que las técnicas mismas de la terapia (Lewelyn \& Hume, 1979, Murphy, Cramer \& Lillie, 1984, Ryan \& Gizynski, 1971, Sloane, Staples, Whipple \& Cristal, 1977, van Beek, 1996, todos ellos en Keijsers, Schaap \& Hoodgduin, 2000), varios estudios se han abocado a estudiar de qué modo los terapeutas cognitivo-conductuales se involucran con el desarrollo y mantenimiento de una buena relación con sus pacientes.

La mayoría de los programas de Terapia Cognitiva Conductual (TCC) proveen al menos alguna agenda para cada sesión terapéutica o cada grupo de sesiones, que anuncia los objetivos y las metas principales de la terapia y el contenido de las sesiones, incluyendo las actividades sugeridas y la tarea (Lewinsohn \& Clarke, 1999). Cabe aclarar que a pesar de que los terapeutas cognitivo-conductuales toman una postura más activa, directiva y de consejo en el tratamiento que terapeutas de otras orientaciones, la misma al ser comparada a otros modos de respuesta tales como las

Tabla 1. Conductas asociadas a directividad

\begin{tabular}{|c|c|c|c|c|}
\hline \multicolumn{5}{|c|}{ Conductas asociadas a directividad } \\
\hline & Por varios autores & $\begin{array}{c}\text { Por algunos autores y que causan } \\
\text { controversia }\end{array}$ & & Por un solo autor \\
\hline - & Direccionamiento del cliente & Confrontación al cliente & $\bullet$ & Expresión de emociones \\
\hline • & Actuación autoritaria & & • & Animado \\
\hline • & Estructuración de la entrevista & & • & Sincero \\
\hline • & Organización & & • & Profundo \\
\hline • & Uso de interrogantes específicos & & • & Descuidado \\
\hline • & Presentación de opiniones "poderosas" & & • & Claro \\
\hline • & Dar consejo & & & \\
\hline • & Modelar conducta & & & \\
\hline • & Decisión & & & \\
\hline • & Sistematicidad & & & \\
\hline • & Retroalimentación & & & \\
\hline • & Actividad & & & \\
\hline • & Asignación de tareas & & & \\
\hline
\end{tabular}


oraciones empáticas y los reconocimientos, aun constituye una pequeña proporción de la conducta verbal total del terapeuta durante el tratamiento.

Datos similares son encontrados por Brunink \& Schroeder (1979 en Keijsers, Schaap \& Hoodgduin, 2000) quienes indican que la TCC utiliza más guía directa que el psicoanálisis, pero menos que la terapia guestáltica. Los mismos terapeutas cognitivos-conductuales reportan emplear significativamente una orientación más directa, educativa y estructurada, que los terapeutas con otras orientaciones como la psicodinámica, humanista o ecléctica. Varios resultados significativos indican que comparados con otras orientaciones terapéuticas, los terapeutas conductuales en general hablan más, hacen más preguntas, son más directivos, ofrecen una mayor estructura y dan información y consejo con mayor frecuencia (Keijsers, Schaap \& Hoodgduin, 2000).

Greenwald, Kornblith, Hersen, Bellack \& Himmeloch (1981), en un estudio del tratamiento de mujeres con depresión unipolar, evidenciaron que los terapeutas cognitivos conductuales realizaron significativamente más intervenciones directivas, registraron más directividad en la medida global de estilo terapéutico, mostraron más iniciativa en las sesiones, hicieron más comentarios no-directivos y mantuvieron mejor clima terapéutico y mayor iniciativa al establecer un clima de apoyo en comparación con los terapeutas dinámicos, evaluando la directividad a través de la conducta del terapeuta: tales como estilo directivo o número de número de intervenciones no-directivas y directivas.

Con relación a la psicoterapia psicodinámica a corto plazo Svartberg \& Stiles (1991), en un metaanálisis, revisaron 19 estudios clínicamente relevantes publicados entre 1978 y 1988 que evaluaban los resultados y efectos diferenciales de la psicoterapia psicodinámica a corto plazo en comparación con el no-tratamiento y con psicoterapias alternativas (en su mayoría cognitivo-conductual, conductual y experiencial) de acuerdo a ciertas características del paciente, del terapeuta y de la terapia. Las características de la psicoterapia psicodinámica a corto plazo se condensaron en tres dimensiones principales, una de las cuales involucraba el estilo del terapeuta (interpretación de la transferencia vs. directividad-interpretación). La técnica de interpretación de la transferencia resulto más efectiva que la técnica directiva en la mayoría de los estudios.

Para los seguidores de la terapia centrada en el cliente la no-directividad es una actitud hacia el proceso de cambio que restringe a los terapeutas de hacer ciertas intervenciones que están intencionalmente formuladas. Se teme que una relajación en esta actitud probablemente conducirá al terapeuta a asumir una pericia que es inconsistente con la teoría de base. Estos terapeutas reconocen que su teoría influencia a los clientes, pero de un modo muy diferente de la que se deriva de las intervenciones directivas (Merry \& Temaner, 2002). También la terapia interpersonal y la psicoterapia psicoanalítica prescinden de instrucciones conductuales directas, siendo estos abordajes indicados para aquellos quienes responden más favorablemente al estímulo discreto o la experimentación (Apple, 1999) ya que se maximiza el involucramiento y la iniciativa del cliente sin directivas conductuales claras.

¿Cual de todos los modelos planteados es mejor? ¿Directivo o no-directivo? La investigación existente no responde unánimemente esta cuestión. Como un dato interesante cabe indicar que en una encuesta realizada en el año 1992 a 75 terapeutas reconocidos (Kleinke, 1995) sobre las futuras direcciones para la psicoterapia, se concluyó que en la próxima década, o sea la que actualmente se encuentra finalizando, la psicoterapia sería menos directiva, más psicoeducativa, centrada en el presente, centrada en el problema y más breve.

\section{Fases de la terapia}

Otra dimensión de importancia en relación a la directividad es el momento de la terapia en que terapeuta y paciente se encuentran trabajando. La aplicación y dosificación de intervenciones directivas debe adecuarse al momento y al nivel de raport alcanzado en las distintas fases del tratamiento.

Según Turkat (1986, en Schaap et al., 1993) durante el primer contacto la directividad en un grado óptimo incrementará la estructura para el cliente y la probabilidad de cooperación. En un sentido contrario a este planteamiento, Bados y García (2001) indican que durante la fase inicial de la intervención $\left(1^{\mathrm{a}}, 2^{\mathrm{a}}\right.$ sesión) la directividad no es conveniente. Una solución a esta diferencia de opiniones es la planteada por Ivey e Ivey (2007) quienes indican que si el terapeuta elige utilizar la directividad, existe la posibilidad de que esta habilidad no funcione por diversos motivos, en ese caso recomienda regresar a la escucha y utilizar una habilidad diferente hasta considerar pertinente retomar una postura directiva.

Existe un mayor consenso en que cuanto más avanzada la terapia, más debe animarse al cliente a tomar la iniciativa (planear el orden del día y las actividades entre sesiones) y a hacer las cosas por sí solo (Bados y García, 2001).

Al analizar desde una perspectiva longitudinal cómo evoluciona la presencia de conductas directivas a lo largo del proceso psicoterapéutico, Keijsers, Schaap, Hoodgduin \& Lammers (1995), estos concluyen que la directividad y la no-directividad pueden combinarse para obtener resultados favorables dependiendo de la fase de tratamiento. Otros resultados bastante en concordancia con los anteriores indican que el control y la directividad dan cuenta del 4\%-1\% de la conducta total del terapeuta durante la primera y la cuarta sesión (Barkham y Shapiro, 1986, Hill et al., 1983, ambos en Schaap et al., 1993). Bachelor \& Salame (2000) intentan estudiar el curso de diferentes facetas de la alianza 
terapéutica a través de la terapia, desde la perspectiva de los participantes en ésta, encontrando que la directividad del terapeuta fue una de las dimensiones de la alianza que demostró efectos significativos en el tiempo en las clasificaciones combinadas de clientes y terapeutas, sin embargo, al analizar por separado a ambos, los resultados no fueron significativos. Se encuentra además que la directividad del terapeuta pareciera particularmente susceptible a las diferentes percepciones individuales y más sensitiva a factores contextuales, como el contenido de la sesión o el trabajo terapéutico específico.

\section{Motivo de Consulta}

A pesar de los fundamentos teóricos que avalan la importancia de la investigación sobre la aplicación de la directividad según el tipo de problema a ser abordado en la terapia, pocos estudios se han centrado específicamente en esta temática.

Se sugiere que a mayor gravedad del cuadro y menor grado de autocontrol por parte del cliente es necesaria una mayor directividad (Bados \& García, 2001). Por ejemplo, con los clientes suicidas el principal objetivo es ayudar a la persona a mantener su vida a pesar de que ésta no vea razones para ello (Ruiz, 1998).

En este marco, Chase (2000), en un estudio de caso único con historia de múltiples hospitalizaciones psiquiátricas debido a recurrentes intentos suicidas, ideación suicida constante, depresión, y recuerdos de abuso sexual recurrentes, describe que los acercamientos directivos fueron utilizados para preservar la seguridad del paciente, desafiar sus distorsiones cognitivas no adaptativas, aumentar sus habilidades de afrontamiento, educarla sobre sus trastornos y tratamiento, procesar traumas y traducir insights y entendimiento en la terapia en acciones fuera de la misma.

En cuanto la intervención a pacientes suicidas algunos investigadores asumen que ciertas técnicas son mejores que otras (por ejemplo, intervención directiva versus no-directiva), sin embargo nunca fueron evaluadas estas apreciaciones por medio de la examinación de la relación entre variables de proceso y de resultado. Mishara \& Daigle (1997) evaluaron los efectos del estilo terapéutico rogeriano en las intervenciones telefónicas en dos centros de prevención de suicidio concluyendo que los consejeros que utilizaron este tipo de terapia disminuyeron significativamente la depresión y consiguieron hacer un trato con el paciente antes de finalizar la llamada. Una de las dificultades de este estudio es que al carecer de un formato comparativo es difícil y arriesgado concluir el tamaño del efecto de esta intervención.

En relación al alcoholismo, en un estudio llevado a cabo en sujetos abusadores de alcohol o drogas se encontró que los clientes consideraron las actitudes no directivas de los consejeros de mayor ayuda en el proceso terapéutico (Toma, 2000).

Carno (1988) examinó el impacto del emparejamiento entre cualidades específicas del paciente y de la terapia en el resultado del tratamiento del alcoholismo. Aunque ninguno de los emparejamientos fue predictor de cambio durante el tratamiento, dos efectos emparejados predijeron cambios en el uso de alcohol en los 6 meses de seguimiento, siendo estos el deterioro funcional con la intensidad del tratamiento y la reactancia del paciente con la directividad de la terapia.

Dada la escasez y la dificultad de un estudio comparativo entre estos estudios, el concluir en una idea general generando prescriptivas para el terapeuta, resulta en extremo difícil y arriesgado.

\section{La directividad como característica particular del terapeuta y del paciente}

Un gran número de estudios naturalísticos y post hoc sobre la directividad del terapeuta (Beutler, Dunbar, \& Baer, 1980, Elliot, Barker, Caskey \& Pistrang, 1982, Hill, Carter \& O'Farrell, 1983, Luborsky et al., 1980, McLellan, Woody, Luborsky \& Goehl, 1988, todos ellos en Bergin \& Garfield, 1994) notaron una preponderancia de las relaciones positivas entre la directividad del terapeuta y los resultados benéficos. Sin embargo, es posible que niveles moderados de directividad faciliten los resultados, mientras niveles altos no. La ausencia de medidas de la directividad, así como que diferentes tipos de clientes reaccionen diferencialmente a terapeutas que son o no altamente directivos impide concluir sobre esto.

Estos hallazgos han conducido a varios investigadores a buscar las cualidades de los clientes que presagian el uso diferencial de intervenciones directivas y evocativas (Beutler, 1991; Beutler, 1983, Shoham-Salomon, Avner \& Neeman, 1989, Shoham-Salomon \& Hannah, 1991, todos ellos en Bergin \& Garfield, 1994).

A continuación se indican algunas de estas investigaciones centradas preferentemente en las cualidades de los clientes.

\section{Libertad de elección percibida y reactancia}

Un buen inicio de la terapia ocurre cuando el terapeuta intenta acomodar su acercamiento a la gravedad del problema y al nivel de reactancia del paciente (Beutler y Clarkin, 1990). Lazarus (1993) establece que las dos variables principales del cliente que guían su postura interpersonal son su percepción de la disposición para el cambio y del nivel de reactancia del cliente.

La reactancia es un concepto similar al de resistencia, aunque la primera se refiere a un estado motivacional, mientras la segunda se refiere a acciones específicas en una situación particular (Jahn \& Lichstein, 1980 en Dowd \& Swoboda, 1984). Muchos investigadores (Basch, 1982, 
Horney, 1942, Jung, 1954, Redl, 1966, Strupp, 1973, todos citados en Bischoff y Tracey, 1995) ven la resistencia al tratamiento influenciada por variables inherentes a la interacción terapéutica, tal como la percepción de pérdida de la autonomía debido a una influencia extrapsíquica (por ejemplo las indicaciones del terapeuta).

El concepto de reactancia al que nos referimos ha cobrado una importancia relevante con relación a la directividad. Se la define como la tendencia a resistirse ante las sugerencias y directivas de otro, para recuperar la pérdida real o amenazada de libertad de acción (Brehm, 1966 en Carnwath \& Miller, 1989) y como se sugirió previamente se considera como parte del modo natural de reaccionar de la persona a muchos tipos de situaciones, no sólo a las terapéuticas. En su teoría de la reactancia Brehm (1966 en Goldfried y Davison, 1981) indica que cuando un individuo percibe que tiene cierta posibilidad de elección, y que esta posibilidad se ve restringida, amenazada o eliminada por alguna autoridad externa, no sólo experimentará un estado de incomodidad subjetiva, sino que también tenderá de modo activo a restaurar su libertad perdida.

En la asignación inicial de tareas propia de los abordajes conductuales, los terapeutas están evaluando implícitamente la reactancia potencial. Si las tareas son completadas, la reactancia potencial es probablemente baja; si no, es probablemente alta (Dowd \& Swoboda, 1984).

Según este modelo cuando el terapeuta conductual hace sugerencias directas o asigna tareas entre sesiones corre el riesgo de activar la reactancia en el cliente e incrementar el riego de falta de cumplimiento. Una persona que es alta en reactancia potencial estaría inclinada a resistir o negar los intentos del terapeuta para controlar o dirigir el curso de la terapia (Dowd \& Swoboda, 1984). Entonces el uso de técnicas paradójicas se convierte en una buena opción. Lo importante es considerar dos factores de principal importancia al evaluar su uso en una determinada situación terapéutica: la reactancia potencial del individuo y la libertad percibida de la conducta problemática.

En resumen, los terapeutas deben acomodar su directividad al nivel de reactancia del cliente, ya que los clientes abiertos a cambiar generalmente se sienten cómodos con un terapeuta probativo y los clientes reactivos o reacios al cambio se beneficiarán más de ser abordados de una manera menos intrusiva, al igual que aquellos pacientes que no ingresan en terapia por iniciativa propia. El emparejamiento de las modalidades terapéuticas y las cualidades del terapeuta a las disposiciones de personalidad del cliente permitirán así maximizar la ganancia terapéutica (Abramowitz, Abramowitz, Roback y Jackson, 1974).

En los estudios clínicos realizados para evaluar el impacto de estas variables del paciente en la terapia los resultados son diversos. Por ejemplo, Bischoff y Tracey (1995) analizan la relación entre la conducta resistente del cliente y la conducta directiva del terapeuta, en una muestra de archivos de sesiones terapéuticas. Sus datos ofrecieron apoyo (aunque modesto) a la hipótesis de que la conducta directiva del terapeuta incrementa la probabilidad de una subsiguiente conducta de resistencia (operacionalizada esta como toda conducta del cliente que muestre su oposición a participar en las tareas terapéuticas dadas por el terapeuta) y de que una conducta no directiva incrementa la probabilidad de una subsiguiente conducta cooperativa del cliente. En una línea similar, Patterson y Forgatch (en Bischoff y Tracey, 1995) en un estudio sobre el cumplimiento de las madres de hijos adolescentes en la terapia familiar conductual encontraron que la directividad del terapeuta interfería con el cumplimiento del cliente incrementando su conducta resistente.

Los hallazgos de Miller, Gayle \& Tonigan (1993) indican que un estilo directivo confrontacional genera significativamente más resistencia en los clientes, prediciendo pobres resultados tras un año. Los autores investigaron el impacto que tiene el estilo del consejero en la motivación al cambio en problemas de alcoholismo. Como intervención directiva, los entrevistadores enfatizaron la evidencia de los problemas derivados del alcohol, dieron advertencias directas y expresaron su desacuerdo cuando el cliente minimizaba sus problemas.

Karmo, Beutler \& Harwood (2002) examinaron las interacciones entre los atributos del paciente y las intervenciones terapéuticas tanto de la TCC como de la terapia sistémica familiar en los resultados de un tratamiento de sujetos alcohólicos. La reactancia del paciente y la directividad del terapeuta fueron predictores importantes del uso del alcohol durante la fase de tratamiento. Los resultados indicaron que los pacientes con alta reactancia tienen mejores resultados en su problema de bebida cuando se les trata con una terapia no directiva, y pacientes con baja reactancia obtienen un mejor resultado con una terapia más directiva. De igual forma los resultados sugieren que en los primeros estadios la directividad del terapeuta puede tener un efecto moderado en el nivel de reacción del paciente en respuesta al tratamiento.

\section{Locus de control}

Los estudios presentados indican que el estilo directivo debe ajustarse no sólo al tipo de problema sino también a otras variables de personalidad del paciente, como nivel de autonomía percibido o locus de control (Karmo, Beutler \& Harwood, 2002; Miller, Gayler \& Tonigan, 1993). Así como ya fue expuesto el hecho de la existencia de diferencias obvias entre quienes aplican la psicoterapia, también es dudoso que todos los pacientes reaccionen similarmente a las terapias pues estas requieren diferentes niveles de involucramiento, participación, y control de la situación por parte del individuo.

Según Bergin \& Garfield (1994) hay algunos tentadores hallazgos que sugieren que una dimensión particular del 
cliente, el estilo de afrontamiento interno vs. externo, puede interactuar con el tipo de tratamiento.

Un concepto estrechamente relacionado al de estilo de afrontamiento es el de locus de control. Bischoff \& Tracey (1995) sugiere que esta podría tener relevancia para la experiencia del cliente de un terapeuta directivo y en la motivación para seleccionar una respuesta resistente como no resistente. El concepto de locus de control fue acuñado por Rotter (1966) y se define por el grado en el cual un individuo cree que los eventos que ocurren en su vida son el resultado de sus propias iniciativas (control interno) o están determinados por la suerte o fuentes de poder externas (control externo).

De acuerdo a lo expuesto entonces podría esperarse que los sujetos con orientación externa fueran terapéuticamente más responsivos que los internos a los tratamientos que prescriben un rol relativamente más activo y poderoso por parte del terapeuta. A la inversa, un abordaje menos directivo, donde el terapeuta tienda a abdicar su autoridad al cliente, debería ser más benéfico para los internos (Abramowitz et al., 1974; Bergin y Garfield, 1994).

Dos estudios empíricos pioneros en el tema son los de Abramovitz et al., (1974) y Friedman y Dies (1974). Abramovitz realiza un estudio en el que explora el resultado de la terapia grupal en un diseño cliente por modalidad en estudiantes universitarios con distrés moderado. Para la evaluación de resultados se usaron diez medidas diferentes, $\mathrm{y}$ en seis de ellas las puntuaciones de sujetos con locus de control externo asignados a uno de los grupos directivos fueron mejores que aquellos con el mismo locus de control pero asignados al grupo no-directivo; y en ocho de estas medidas los sujetos con locus de control interno en el grupo no-directivo mostraron mejores resultados que los sujetos con locus interno de los grupos directivos.

En el mismo año otro estudio (Friedman y Dies, 1974) examinaba las respuestas diferenciales de estudiantes con ansiedad frente a los exámenes que diferían en la Escala de Locus de Control Interno-Externo de Rotter a tres formas de terapia aplicadas durante cinco semanas: desensibilización sistemática, counseling orientado a la discusión y desensibilización automatizada. Los resultados mostraron que los sujetos con orientación externa que recibieron counseling o desensibilización sistemática sintieron que ellos mantuvieron demasiado control de la terapia, mientras los internos generalmente indicaron la percepción de una cantidad opcional de control en el counseling. También se les preguntó sobre las alteraciones que habrían hecho en sus terapias y de las respuestas dadas se concluyó que a pesar de las condiciones del tratamiento los sujetos con locus de control interno habrían elegido significativamente "más control del cliente" que los sujetos con locus de control externo. Sin embargo estos últimos aprovecharon mejor la oportunidad de individualizar la terapia incluyendo ítems de su propia elección en las jerarquías de la desensibilización.
En función de dos criterios se pudo corroborar la predicción de que los sujetos con locus de control interno mostrarían mayor resistencia al control implicado en las dos terapias conductuales que los sujetos con orientación externa.

Por último Jones \& Gelso (1988) encuentran que individuos que son dogmáticos y con un locus de control externo tenderían a esperar recursos externos que sean responsables de su destino. En la consulta estos clientes pueden querer, necesitar o esperar que el terapeuta sea una autoridad, provea las respuestas y tome las responsabilidades de lo que sucede en la terapia. También plantean que a menudo estos clientes se presentan como resistentes al cambio.

Aun resulta complejo entender y explicar la naturaleza, fuerza y generalidad de la aparente interacción entre directividad del tratamiento y reactancia del cliente baja vs. alta, y foco del tratamiento y estilo de afrontamiento internoexterno del cliente, por lo que se requiere más investigación para lograr un concepto unívoco sobre el tema.

\section{Características cognitivas}

En relación al desarrollo y estado cognitivo de los sujetos, los estudios sugieren que en el caso en que se presenta resistencia o incumplimiento de las tareas debido a que se carece de las destrezas necesarias para ejecutar las peticiones del terapeuta (por ejemplo tareas de aserción) el problema podría sortearse proporcionando una intervención directa (Cormier \& Cormier, 1994). Asimismo en pacientes dispuestos a ser dirigidos, motivados principalmente para alcanzar metas específicas, pacientes gravemente perturbados y frágiles o que se encuentren en crisis se recomienda un tipo de acercamiento directivo (Kleinke, 1995) que se utilizará en tanto resulte terapéuticamente productivo siendo la meta última que el paciente se independice por completo del terapeuta (Goldfried \& Davison, 1981).

Jones (1997), en un estudio con estudiantes postsecundarios, encontró que en términos de preferencias pre-counseling, aquellos estudiantes con bajos niveles de desarrollo intelectual y social-cognitivo tenían una preferencia más fuerte por la directividad y pericia del consejero.

También se pueden extraer resultados relevantes a partir de la investigación en terapias muy específicas. Por ejemplo en un estudio sobre el tratamiento de fluidez de inteligencia en personas mayores los resultados avalan la utilización de la terapia cognitivo conductual para estos casos. Además afirman que un estilo no directivo de actividad exploratoria abierta tendría un obstáculo ya que requiere pacientes que puedan abstraer significados e implicaciones del dialogo terapéutico (Doubleday, King \& Papageorgiou, 2002), actividad que puede sufrir cierto deterioro a medida que avanza la edad. 


\section{Características sociodemográficas}

Se ha planteado que variables tales como género, edad y origen étnico pueden influenciar la interacción terapéutica (Bischoff y Tracey, 1995).

En relación al sexo del terapeuta, Shields \& McDaniel (1992) presentan un estudio realizado en entrevistas iniciales de terapia estratégico-estructural en familias con niños con problemas. Sus resultados evidenciaron que los terapeutas hombres son verbalmente más activos que las mujeres y que interrumpen más a menudo, además que tienden a ser más instrumentales y directivos que las terapeutas mujeres.

Respecto a la acción de estas variables en el paciente se ha reportado que los pacientes mayores clasifican a sus terapeutas mas favorablemente y los terapeutas mayores son clasificados por sus pacientes como siendo más directivos (Bennun \& Schindler, 1988). Al evaluar a varones con respecto a su preferencia de dos tipos de counseling de orientación profesional contrastantes, manifestaron en su mayoría preferencia por un acercamiento directivo más que un acercamiento de orientación contextual y emocional (Rochlen \& O'Brien, 2002). Datos similares son encontrados en una muestra de pacientes de un hospital alemán para pacientes neuróticos y psicosomáticos, y en el intento de evaluar la conducta percibida tanto por el terapeuta como por el cliente, se encontró que a mayor edad de los terapeutas estos eran percibidos como más y se estableció además una asociación entre la percepción de la conducta del terapeuta y del paciente y los resultados del tratamiento (Bennun et al., 1986).

En otro estudio los estudiantes jóvenes mostraron expectativas más positivas respecto a la terapia comparados con los adultos y esperaron a consejeros con mayor capacidad de aceptación, directivos, abiertos, expertos y tolerantes (Wagner, 1999).

A partir de la investigación en la relación médico-paciente se ha señalado que personas más jóvenes con mayor nivel educacional prefieren tomar un rol activo en la decisión médica, mientras los hombres, separados o divorciados y pacientes con un severo pronóstico tienden a preferir un rol más pasivo y permiten al médico tomar más decisiones (Cassileth, 1980, Ende, 1989, Kapp, 1991, todos citados en Kaplan, Schneiderhan, Harrow \& Omens, 2002).

Las características étnicas de los usuarios es otro factor estudiado. En un estudio realizado en estudiantes de Singapur y estadounidenses acerca de sus expectativas sobre el counseling y la percepción de efectividad del consejero en los estilos directivo y no directivo, se notó que los estudiantes de Singapur en particular y los hombres en general de la muestra, esperaron que el consejero fuese más directivo, empático y se auto-revelase más (D'Rosario, 1996).

Merta, Ponterotto \& Brown (1992) en un estudio que compara la efectividad de dos estilos directivos de consejería académica (autoritario y colaborativo) en estudiantes extranjeros (asiáticos) encuentran que en estudiantes con una alta aculturación es más efectivo un estilo autoritario, mientras que en estudiantes con baja aculturación es mas efectivo un estilo colaborativo (definiendo el nivel de aculturación a través de dos indicadores: tiempo en el país y autopercepción en el manejo del inglés). Otras investigaciones sugieren que sujetos asia-americanos tienden a preferir una aproximación estructurada y directiva de counseling (Atkinson, Maruyama \& Matsui, 1978, Brown et al., 1973, Sue, 1981, todos citados en Akutsu, Lin \& Zane, 1990). Pero los resultados hallados por Cheng (2000) no corroboran la visión que se tiene desde la literatura que concluye que los asiáticos y los asiáticos-americanos prefieren en la psicoterapia los estilos directivos a los no-directivos. En su estudio dirigido a observar las preferencias de estudiantes universitarios chinos y chinos-americanos respecto a tres tipos de terapia diferentes, los participantes prefirieron en primer lugar el acercamiento psicoanalítico, seguido de la terapia centrada en el cliente y de la terapia conductual.

\section{Expectativas y satisfacción con la terapia}

Las personas inician la terapia con ciertas expectativas, las cuales se relacionan estrechamente a la efectividad de la terapia, si el estilo del terapeuta y/o el abordaje empleado difieren marcadamente de las expectativas del paciente, los resultados positivos son improbables (Lazarus, 1993).

Respecto a las expectativas iniciales del cliente Tinsley, Bowman \& Westcot (1993) evalúan en una muestra de consejeros psicológicos la percepción que tienen acerca de las expectativas de sus clientes sobre la consejería y la psicoterapia. Una mayoría (88\%) percibió de sus clientes expectativas irrealmente altas sobre directividad; se hipotetiza que este patrón de expectativas irreales podría mediar el proceso terapéutico provocando efectos en detrimento del proceso de consejería, cuando no se cumplen los deseos del cliente.

Navarro, et al., (1987a) estudió las expectativas que el paciente tiene respecto a la forma en que debiera comportarse su terapeuta. Propone que tenerlas en cuenta desde el inicio de la terapia podría favorecer la adherencia y la motivación de la persona. Estos autores encontraron que los pacientes consideran más deseables las características del terapeuta que están relacionadas con hábitos de estructuración y claridad, en comparación con otros que expresan simpatía e interés en el cliente. Además reportan una relación significativa y positiva entre edad y directividad, los varones puntuaron más alto que las mujeres en este factor. Directividad correlacionó también de forma positiva y significativa con las variables diagnóstico, aquellos con problemas de dependencia puntuaron más que el resto, número de semanas de tratamiento y la duración de este en conjunto, así como el grado en que los sujetos se muestran satisfechos con la terapia. 
En otro estudio relacionado con expectativas sobre el terapeuta, Navarro, et al., (1987b) evalúan la conducta de psicoterapeutas de diversas orientaciones teóricas con autoinformes, asi como con sus pacientes. Al comparar los resultados obtenidos en los instrumentos se observa que los pacientes consideran que un terapeuta ideal debería presentar en mayor medida conductas que indiquen apoyo y directividad, mientras que los terapeutas reales eligen con mayor frecuencia alternativas de respuesta que expresan exploración y empatía. Al contrastar puntuaciones sólo se dan diferencias significativas en las puntuaciones de Directividad, los pacientes perciben a sus terapeutas de una forma menos directiva (en el sentido de estructuración y claridad), de lo que piensan que debería ser un terapeuta ideal.

Como conclusión los pacientes se imaginan que un "buen" terapeuta debiera mostrar preferentemente las cualidades de directividad y apoyo y las características de directividad (claridad, estructuración).

En sus conclusiones los autores destacan que son precisamente las variables preferidas por los clientes las que se muestran relacionadas de forma positiva con la consecución de los resultados deseados en el tratamiento, lo que parece bastante claro desde las fases iniciales de la terapia.

Otra arista relacionada con las expectativas es la intención de utilización, es decir, la motivación hacia tomar terapia podría estar basada fundamentalmente en las expectativas que la personas estudiada tiene sobre como debe ser la terapia o el que la brinda (Akutsu, et al., 1990; Moore \& Kenning, 1996) .

\section{Discusión}

Varios años de investigaciones han dado a conocer que la calidad de la relación terapéutica puede predecir resultados y que las percepciones y expectativas que de la misma tienen los agentes implicados son de suma importancia. Un punto de partida a modo de conclusión, puede centrarse en la propuesta de que "las relaciones de elección" (como las ha llamado Lazarus, 1993) que uno establece en la terapia no son menos importantes que las "técnicas de elección" para un resultado efectivo. O sea que un repertorio flexible de estilos relacionales, más un amplio rango de técnicas pertinentes parecen aumentar los resultados del tratamiento.

En respuesta a los argumentos sobre la confusa multiplicidad de los factores intervinientes que dificultan el estudio y la capacidad de discriminar las variables relevantes en el marco de las habilidades terapéuticas, se ha establecido que la directividad constituye de hecho una de las dimensiones importantes en el área, consecuentemente ha sido necesario determinar las conductas específicas que son indicadores de los términos directivo y no directivo.

Los terapeutas necesitan evaluar en qué situación, considerando factores como el estadio de la terapia y las condiciones de consulta del sujeto, y con qué clientes, teniendo en cuenta el tipo del disturbio y las expectativas y características del cliente, conductas terapéuticas particulares producirán con mayor probabilidad resultados positivos.

Aún antes de que se inicie el tratamiento los pacientes tienen una percepción definida del terapeuta al igual que una concepción determinada de lo que esperan de la terapia. El hecho de que el paciente se sienta cómodo con una terapia directiva o no-directiva puede variar de persona a persona. Es decir, la preferencia y conveniencia de un estilo directivo en la terapia dependerá de diversos factores que van desde la fase de la terapia y el momento del curso de la enfermedad en la que se encuentre el cliente hasta las percepciones y características de personalidad del sujeto, pasando por el problema que motiva la consulta o diversas particularidades socio-demográficas tanto del paciente como del terapeuta.

En el ámbito clínico es posible comprobar que a diferencia de las conductas conflictivas que entorpecen el curso del tratamiento, otras conductas lo facilitan, es el caso de pacientes cuyas características, que pueden representar o no el motivo por el que consulta, lo hacen sensible a la influencia del terapeuta. Sin embargo, y al igual que en el tratamiento de cualquier persona con problemas psicológicos se aconseja ser lo menos directivo que las condiciones lo permitan, ya que el objetivo último será promover el aprendizaje de los recursos necesarios para alcanzar un funcionamiento autónomo y satisfactorio en el cliente tanto para él mismo como para su entorno.

Los autores acuerdan con la afirmación de que la pregunta no debe ser si las declaraciones directivas, las explicaciones o los consejos del terapeuta favorecen el alcance por parte del paciente de cambios conductuales, sino en qué momento o bajo cuáles circunstancias estos son aceptados y compartidos por los pacientes (Keijsers et al., 1995). También Lazarus (1993) acuerda en este aspecto al afirmar que las decisiones con respecto a adoptar diferentes posturas en la relación terapéutica incluyen cuándo y cómo ser directivo, dar soporte, ser reflexivo, frío, cálido, tibio, formal o informal.

En un intento de organizar los hallazgos más relevantes sobre el tema de modo que puedan servir a los clínicos en su actuación terapéutica se presenta un resumen de las ideas y propuestas generales a partir del análisis de los estudios revisados (Tabla 2)

Los datos aportados por la literatura sobre directividad no son del todo concluyentes. Las limitaciones metodológicas de las investigaciones no hacen posible extraer conclusiones generalizables a todos los procesos terapéuticos independientemente de las características particulares del terapeuta y de la persona que requiere ayuda, tales como edad, sexo, personalidad, expectativas u orientación, ni de las particularidades del problema que motiva la consulta, entre otras. 
Tabla 2. Grado de directividad sugerido con base en la evidencia

\begin{tabular}{|c|c|}
\hline ¿Cuando ser directivo? & Referencia \\
\hline Pacientes con locus de control externo & $\begin{array}{l}\text { Abramovitz et al., } 1974 \\
\text { Friedman y Dies, } 1974 \\
\text { Bergin y Garfield, } 1994 \\
\text { Jones y Gelso, } 1988\end{array}$ \\
\hline Pacientes suicidas, en crisis o con cuadros graves & $\begin{array}{l}\text { Mishara y Daigle, } 1997 \\
\text { Ruiz, } 1998 \\
\text { Bados y García, } 2001 \\
\text { Kleinke, } 1995\end{array}$ \\
\hline Pacientes alcohólicos & Toma, 2000 \\
\hline Pacientes alcohólicos no reactantes & Karmo, Beutler y Harwood, 2002 \\
\hline Personas mayores o con problemas cognitivos & Doubleday, King y Papageorgiou, 2002 \\
\hline Pacientes con pronóstico severo & Kaplan et al., 2002 \\
\hline Personas con menor grado de autocontrol & Bados y García, 2001 \\
\hline Personas sin destrezas necesarias para ejecutar las tareas asignadas por el terapeuta & Cormier y Cormier, 1994 \\
\hline Varones & Rochlen y O’Brien, 2002 \\
\hline Algunas minorías étnicas, en proceso de adaptación cultural & $\begin{array}{l}\text { D'Rosario, } 1996 \\
\text { Merta, Ponterotto y Brown, } 1992\end{array}$ \\
\hline Personas con bajo nivel de desarrollo intelectual y social-cognitivo & Jones , 1997 \\
\hline ¿Cuando no ser directivo? & Referencia \\
\hline Fase inicial de la Terapia $\left(1^{\circ}, 2^{\circ}\right.$ sesión $)$ & Bados y García, 2001 \\
\hline Pacientes con alta reactancia & $\begin{array}{l}\text { Dowd y Swodowa, } 1984 \\
\text { Karmo, Beutler y Harwood, } 2002\end{array}$ \\
\hline Pacientes con locus de control interno & $\begin{array}{l}\text { Abramovitz et al., } 1974 \\
\text { Friedman y Dies, } 1974 \\
\text { Bergin y Garfield, } 1994\end{array}$ \\
\hline Personas jóvenes & Kaplan et al., 2002 \\
\hline Personas con mayor nivel educacional & Kaplan et al., 2002 \\
\hline
\end{tabular}

Una gran limitación de la literatura tiene relación con la demarcación del concepto de directividad. Los problemas derivados de la falta de una definición unívoca se expresan también en la diversidad, aunque no multiplicidad, de instrumentos de evaluación de la directividad. El establecimiento de conductas operacionales claramente observables se encuentra metodológicamente vinculado a la forma de evaluarlas. Además se propone como necesario considerar en la evaluación de la directividad la percepción del sujeto de las intervenciones, es decir, considerar la presencia de conductas catalogadas objetivamente como directivas y si éstas son significadas o vivenciadas por el paciente como directivas o no.

Además de las dos grandes limitaciones mencionadas que están a la base de la literatura revisada, los resultados encontrados presentan otras características que imposibilitan el extraer conclusiones certeras y definitivas, siendo estas:

a) Muchos de los estudios se han realizado con muestras reducidas.

b) Muchos de los estudios, especialmente los relacionados con expectativas, se han realizado preferentemente en personas sin patologías, principalmente con muestras conformadas por estudiantes universitarios que suelen presentar niveles de distrés moderados, cuadros sub-clínicos o ausencia total de indicadores clínicos.

c) Se han estudiado sólo algunas patologías y su relación con la directividad, por lo tanto no se conoce si la variable diagnóstico es un factor relevante en el tema, y si lo fuera, si distintos cuadros psicopatológicos presentan respuestas diferenciales ante enfoques directivos o no-directivos.

d) No se ha encontrado en la literatura revisada investigaciones que den cuenta acerca de la directividad concebida como un rasgo de personalidad, y su influencia en las conductas del terapeuta y por ende en la relación terapéutica.

e) Los tratamientos que se aplican son relativamente cortos y probablemente no permitan evaluar bien qué sucede con las intervenciones directivas más allá de la sesión número 10 o 13, y cuál es el punto óptimo de directividad en la fase final del tratamiento y en el seguimiento.

f) Si se concibe la directividad no como un concepto polar (directivo/ no-directivo) sino como un continuo con diversos grados es viable pensar que los resultados poco consistentes entre estudios se deba a que en cada uno de ellos se aplicaron grados y/o modalidades diferentes de intervenciones directivas. 
De estas limitaciones es posible derivar una serie de propuestas necesarias para ajustar la investigación en el tema de la directividad.

a. Con respecto a los diseños son necesarios estudios multifactores, multipatologías, multiestilos que permitan establecer conclusiones sobre la relación entre varios factores. Como ha sido presentado, las variables que pueden contribuir a una mejor o peor respuesta al tratamiento al interactuar con la directividad, han sido estudiadas por separado y en la actualidad muy pocos estudios han logrado sacar conclusiones claras sobre la relación entre más de dos factores. Por ejemplo, cabría pensar que el nivel de reactancia y el locus de control tienen alguna relación pues ambos son dimensiones del modo de respuesta del sujeto a las demandas ambientales, sin embargo poco o nada se sabe de la naturaleza de esta relación, menos aún de cómo ésta podría influir en la respuesta del sujeto a la directividad del terapeuta. Muchas otras combinaciones podrían dar resultados en extremo útiles, por ejemplo tipo de problema, momento del curso de la enfermedad y fase de la terapia, o estilos del paciente y del terapeuta y tipo de terapia, etc. b. También se propone la inclusión de otros marcos de análisis en los estudios, como por ejemplo teorías de género, de grupo o de personalidad. Justifica esta propuesta los interrogantes sobre el impacto que la directivas del terapeuta pueden tener en un paciente de acuerdo al sexo de cada uno y considerando como punto de partida que a un nivel, muchas veces no-manifiesto, en el interior de cualquier interacción se establecen relaciones de poder entre los sexos. Por otro lado, a diferencia de la terapia individual, en los grupos la presencia entre los miembros de roles, funciones y jerarquías diferenciales también puede complejizar la elección por el uso de intervenciones directivas. Por último, las teorías de personalidad permitirían investigar la directividad como característica de personalidad del terapeuta.

c. Relacionar los hallazgos obtenidos con el entrenamiento de terapeutas, lo que exigirá conocer las posibilidades de entrenar a personas que no son directivas para serlo cuando las condiciones lo requieren y viceversa, si es posible entrenar a personas directivas a ser menos directivas cuando sea apropiado.

d. Asimismo, son necesarios estudios sobre la edad del terapeuta y el uso de directividad desde dos aristas: a)

Figura 1. Variables relacionadas con la directividad en el proceso terapéutico

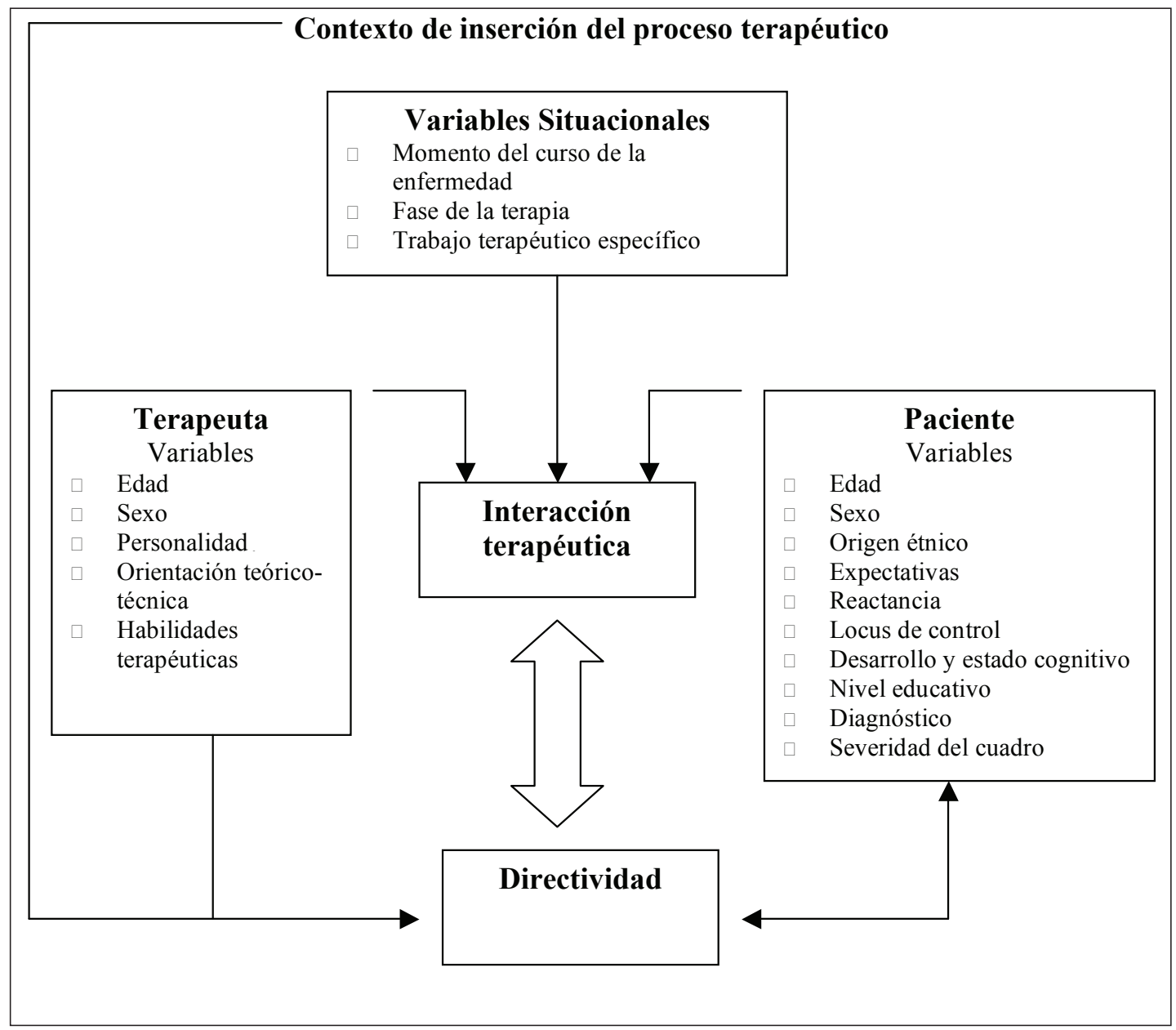


relación entre edad y desarrollo del rasgo de personalidad (si es que se determinase la directividad como un rasgo), y b) relación entre percepción de pericia y aceptación del estilo directivo por parte del paciente, partiendo del supuesto que en una persona creará mas disonancia y resistencia el aceptar instrucciones directivas cuando perciba diferencias de edad significativas o poca experiencia en el terapeuta.

e. Si se reconociese que las características de trastornos específicos interactúan con el modo de reaccionar al estilo terapéutico cabría priorizar los estudios en población con patologías primarias que representen de un modo más confiable a quienes acuden a una consulta psicológica.

f. Por último se sugiere evaluar la eficacia de la directividad en distintos niveles de intervención sanitaria y en distintos contextos asistenciales. Es sabido que entre quienes consultan en servicios de Atención Primaria de Salud la tasa de adherencia es muy baja y la tasa de deserción muy alta, sin sobrepasar en la mayoría de las ocasiones la asistencia a seis entrevistas, por lo que en este contexto podría ser de mayor utilidad una terapia más directiva a fin de lograr resultados significativos en las pocas sesiones que suele asistir un paciente. Otro ejemplo puede ser la intervención en pacientes hospitalizados de corta estadía, donde el accionar debe ser distinto al de pacientes hospitalizados de larga estadía o al de la consulta privada. En la asistencia privada a pesar de que el terapeuta dispone de más libertad para organizar el proceso psicoterapéutico es importante recordar que las expectativas del paciente y la congruencia que las mismas encuentren con la oferta asistencial determinarán en gran medida que el mismo siga asistiendo y trabajando en pos del cambio.

En resumen, a pesar de la diversidad de variables que pueden intervenir en la interacción psicoterapéutica la directividad del terapeuta ya está reconocida como uno de los factores relevantes en dicha interacción. En el uso y la aplicación de las intervenciones directivas es necesario considerar los factores que han sido descriptos a lo largo del trabajo y cuyas fuentes se encuentran básicamente en el sujeto que consulta, en quien atiende la demanda de asistencia y en el contexto de esta interacción (Figura 1).

De los estudios resaltan limitaciones metodológicas así como ciertos recaudos a considerar en la combinación de estas variables, por lo tanto si los lineamientos explicados en el trabajo sobre la aplicación de directividad no son atendidos con la "cautela" suficiente pueden parecerse más a un arriesgado recetario de combinaciones posibles que no llegaría en ninguno de los casos a cubrir las variabilidad infinita de situaciones que pueden presentarse en la clínica diaria. Sin perjuicio al aliento por más investigación, surge una vez más la idea de la psicoterapia como un arte, aunque basado en muchos casos en hallazgos de validez empírica, un arte al fin.

\section{Referencias}

Abramowitz, C.V., Abramowitz, S.I., Roback H., Jackson C. (1974). Differential effectiveness of directive and nondirective group therapies as a function of client internal- external control. Journal of Consulting and Clinical Psychology, 42, 849-853.

Akutsu, P., Lin, C. \& Zane, N. (1990). Predictors of utilization intent of counseling among chinese and white students: A test of the proximaldistal model. Journal of Counseling Psychology, 37, 445 -452.

Apple, R. (1999). Interpersonal therapy for bulimia nervosa. Journal of Clinical Psychology, 55, 715-725.

Bachelor, A. \& Salame, R. (2000) Participants perceptions of dimensions of the therapeutic over the course of therapy. The Journal of Psychotherapy: Practice and Research, 9, 39 - 53.

Bados, A., \& García, E. (2001). Habilidades terapéuticas. Barcelona: Universidad de Barcelona.

Bennun, I., Hahlweg, K., Schindler, L. \& Langlotz, M. (1986). Therapist's and client's perceptions in behavior therapy: the development and cross-cultural analysis of an assessment instrument. British Journal of Clinical Psychology, 11, 275-283.

Bennun, I. \& Schindler, L. (1988). Therapist and patient factors in the behavioral treatment of phobic patients. British Journal of Clinical Psychology, 27, 145-150.

Bergin, A. E. \& Garfield, S. L. (Ed.). (1994). Handbook of psychotherapy and behavior change ( $4^{\mathrm{a}} \mathrm{Ed}$.). USA: John Wiley \& Sons, Inc.

Beutler, L. (1991). Have all won and must all have prizes? Revisting luborsky et al.'s veredict. Journal of Consulting and Clinical Psychology, 59, 226-232

Beutler L \& Clarkin, J. (1990) Systematic Treatment selection. New York: Brunner /Mazel.

Bischoff, M., \& Tracey, T. (1995). Client resistance as predicted by therapist behaviour: A study of sequential dependence. Journal of Counseling Psychology, 42, 487- 495.

Carmo, M.P. (1998). Identifying patient attributes and elements of psychoteraphy that impact the effectiveness of alcoholism treatment. Dissertation Abstracts International: Section B. The Sciences and Engineering, 58, 3926.

Carnwath, T., \& Miller, D. (1989). Psicoterapia conductual en asistencia primaria: manual práctico. Barcelona: Martínez Roca, S.A.

Chase, K. (2000). The integrative stage model approach to the case of Katrina. Cognitive and Behavioral Practice, 7, 489-497.

Cheng, Y. (2000). The influences of chinese and chinese-cmerican students' cultural worldviews and acculturation levels on preferences toward three psychotherapy approaches. Dissertation Abstracts International, Section B. The Sciences and Engineering, 61, 2193.

Cormier, W. \& Cormier, L. (1994). Estrategias de entrevista para terapeutas: Habilidades básicas e intervenciones cognitivo-conductuales. Bilbao. Editorial Desclée de Brouwer.

Doubleday, E., King, P., \& Papageorgiou, C. (2002). Relationship between fluid intelligence and ability to benefit from cognitive-behavioural therapy in older adults: A preliminary investigation. British Journal of Clinical Psychology, 41, 423-428.

D' Rosario, V. (1996). Singaporean and United States college students worldviews, expectations of counseling, and perceptions of counselor effectiveness based on directive and nondirective counseling styles. Disertation Abstracts International, Section A. Humanities and Social Sciences, 56, 2564.

Dowd, E.T. \& Swoboda, J.S. (1984). Paradoxical interventions in behavior therapy. Journal of Behavior Therapy and Experimental Psychiatry, $15,229-234$.

Friedman, M. L., \& Dies, R. R. (1974). Reactions of internal and external test-anxious students to counseling and behavior therapies: Journal of Consulting and Clinical Psychology Vol 42(6) Dec 1974, 921.

Goldfried, M.R. \& Davison, G.C. (1981). Técnicas terapéuticas conductistas. Buenos Aires: Paidos.

Greenwald, D., Kornblith, S. Hersen, M., Bellack, A. \& Himmelhoch, J. (1981). Differences between social skills therapists and psychotherapists 
in treating depression. Journal of Consulting and Clinical Psychology, 49, 757-759.

Ivey, A.E., e Ivey, M.B. (2007). Intentional Interviewing and Counseling. Facilitating Client Development in a Multicultural Society. (6 ${ }^{\mathrm{a}}$. Ed.). Belmont, Ed. Thomson Brooks/Cole

Jones, A., \& Gelso, Ch. (1988). Differential effects of style of interpretation: another look. Journal of Counseling Psychology, 35, 363-369.

Jones, D.A. (1997). Cognitive development and its relationship to expectations and preferences for counselling: A test of two models. Dissertation Abstracts International: Section A. The Humanities and Social Sciences, 58, 1602.

Kaplan, K., Schneiderhan, M., Harrow, M. \& Omens, R. (2002). Autonomy, gender, and preference for paternalistic or informative physicians: A study of the doctor-patient relation. Ethics and Medicine 18, 49-60.

Karmo, M., Beutler, L., \& Harwood, T. (2002). Interactions between psychotherapy procedures and patient attributes that predict alcohol treatment effectiveness: A preliminary report. Addictive Behaviors, 27, 779-797.

Keijsers, G., Schaap, C. \& Hoodgduin, C. (2000). The impact of interpersonal patient and therapist behavior on outcome in cognitive-behavior therapy. Behavior Modification, 24, 264-297.

Keijsers, G., Schaap, C., Hoodgduin, C. \& Lammers, M. (1995). Patienttherapist interaction in the behavioral treatment of panic disorder with agoraphobia. Behavior Modification, 19, 491-517.

Kleinke, C. (Ed.). (1995) Principios comunes en psicoterapia. Bilbao. Editorial Desclée de Brouwer.

Lazarus, A. (1993). Tailoring the therapeutic relationship, or being an authentic chameleon. Psychotherapy, 30, 404-407.

Lewinsohn, P. \& Clarke, G. (1999). Psychosocial treatments for adolescent depression. Clinical Psychology Review, 19, 329-342.

Merry, T. \& Temaner, B. (2002). The nondirective attitude in client-centered therapy: a response to Kahn. Journal of Humanistic Psychology, 42, 66-77.

Merta, R., Ponterotto, J., \& Brown, R. (1992). Comparing the effectiveness of two directive styles in the academic counseling of foreign students. Journal of Counseling Psychology, 39 214-218.

Miller, W., Gayle, R., \& Tonigan, J.S. (1993). Enhancing motivation for change in problem drinking: A controlled comparison of two therapist styles. Journal of Consulting and Clinical Psychology, 61, $3,455-461$.

Mishara, B., \& Daigle, M. (1997). Effects of different telephone interventions styles with suicidal callers at two suicide prevention centers: An empirical investigation. American Journal of Community Psychology. $25,865-881$.
Moore, K.E. \& Kenning, M. (1996). Assessing client satisfaction in a psychology training clinic. Journal of Mental Health Administration, 23, 180-189.

Navarro, A., Schindler, L., \& Silva, F. (1987a). Evaluación de la conducta del psicoterapeuta: preferencias del cliente. Evaluación psicológical Psychological Assessment, 3, 101-123.

Navarro, A., Schindler, L., \& Silva, F. (1987b). Estilos comportamentales del psicoterapeuta y preferencias del cliente. Evaluación psicológica / Psychological Assessment, 3, 409-422.

Rochlen, A.B., \& O'Brien, K.M. (2002). The relation of male gender role conflict and attitudes toward career counseling to interest in and preferences for different career counseling styles. Psychology of Men and Masculinity, 3, 1, 9-21.

Rotter, J. (1966). Generalized expectancies for internal versus external control of reinforcements. Psychological Monographs, 80, Whole No. 609.

Ruiz, M. (1998). Habilidades terapéuticas. En Vallejo, M.A.,(Ed.). Manual de terapia de conducta, (pp.83-131). Madrid: Dykinson

Schaap, C., Bennun, I., Schindler, L., \& Hoogduin, K. (1993). The therapeutic relationship in behavioral psychotherapy. Nueva York: Ed.Wiley.

Shields, C., \& McDaniel, S. (1992). Process Differences between male and female therapist in a first family interview. Journal of Marital and Family Therapy, 18, 143-151.

Svartberg, M. \& Stiles, T. (1991). Comparative effects of short term psychodinamic psychoteraphy: a meta-analysis. Journal of Consulting and Clinical Psychology, 59, 5, 704-714.

Tinsley, H., Bowman, S. \& Westcot, A. (1993). Counseling psychologists' perceptions of the occurrence and effects of unrealistic expectations about counseling and psychotherapy among their clients. Journal of Counseling Psychology, 40, 46-52.

Toma, F. (2000). A comparison of the perception of drug and alcohol measures on the helpfulness of therapeutic attitudes used by counsellors. European Journal of Psychotherapy, Counselling and Health, 3, 103-110.

Wagner, N. (1999). A comparison of adult and traditionally-aged students' expectations about counseling. Dissertation Abstracts International, Section B. The Sciences and Engineering, 59, 5589. 